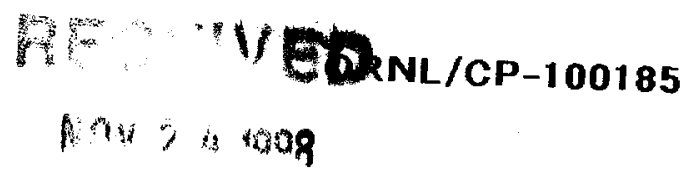

\title{
Proton Decay Studies of the Light Lu, Tm and Ho Isotopes*
}

\author{
J. C. Batchelder ${ }^{1}$, C. R. Bingham ${ }^{2,3}$, C. J. Gross ${ }^{2,7}$, R. Grzywacz ${ }^{3}$,

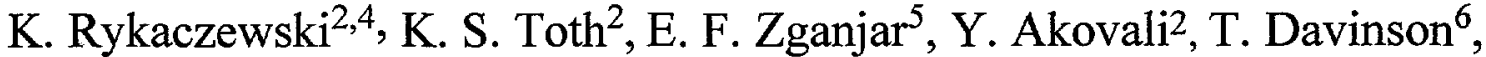 \\ T. N. Ginter ${ }^{7}$, J. H. Hamilton ${ }^{7}$, Z. Janas ${ }^{4}$, M. Karny ${ }^{4}$, S. H. Kim³, \\ B. D. MacDonald 9 , J. F. Mas ${ }^{2}$, J. W. McConnell ${ }^{2}$, A. Piechaczek ${ }^{5}$, J. J. Ressler10, \\ R. C. Slinger ${ }^{6}$ J. Szerypo ${ }^{11}$, W. Weintraub ${ }^{3}$, P. J. Woods ${ }^{6}$, C.-H. Yu ${ }^{2}$ \\ 1 Oak Ridge Associated Universities, Oak Ridge TN, 37831 USA \\ 2 Physics Division, Oak Ridge National Laboratory, Oak Ridge, 37831 TN USA \\ 3 University of Tennessee, Knoxville TN 37996 USA \\ 4 IEP, Warsaw University, 00681 Warsaw, Hoza 69, Poland \\ 5 Louisiana State University, Baton Rouge, LA 70803 USA \\ 6 University of Edinburgh, Edinburgh, EH9 3JZ, United Kingdom \\ 7 Vanderbilt University, Nashville TN 37235 USA \\ 8 Oak Ridge Institute for Science and Education, Oak Ridge, TN 37831 USA \\ 9 Georgia Institute of Technology, Atlanta GA 30332 USA \\ 10 University of Maryland, College Park, MD 20742 USA \\ 11 Joint Institute for Heavy Ion Research, Oak Ridge TN, 37831 USA
}

\begin{abstract}
A double-sided Si-strip detector system has been installed and commissioned at the focal plane of the Recoil Mass Spectrometer at the Holifield Radioactive Ion Beam Facility. The system can be used for heavy charged particle emission studies with half-lives as low as a few $\mu$ sec. In this paper we present identification and study of

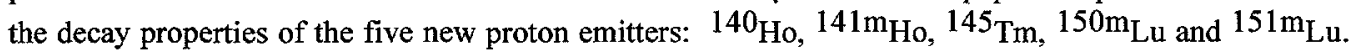

\section{INTRODUCTION}

Nuclei which are energetically unbound to the emission of a proton are located beyond the proton drip line. Observation of protons emitted from these isotopes allows us not only to establish the limits of stability for a given element, but also gives information on the structure and mass of the parent nucleus. Study of the decay of protonemitting isotopes allows one to study nuclear structure effects in nuclei that are inaccessible by means of in-beam experiments. The emitted proton tunnels through the Coulomb and centrifugal barriers, and the decay probability depends strongly on the energy of the proton and on its angular momentum $\ell$. Because of this, the $\ell$ value of the emitted proton can often be determined through the use of a simple spherical WKB calculation of the expected rate of the tunneling process. However, this calculation does not take into account the details of the nuclear structure effects. Therefore, the difference between the calculated and experimental half-lives is due to nuclear structure effects such as an occupation of a respective proton orbital, and is known as the spectroscopic factor. The ratio of the calculated half-life to the measured value is defined as the experimental spectroscopic factor [1]:

"The submitted manuscript has been authored by a contractor of the U.S. Govemment under contract No. DEAC05-960R22464. Accordingly, the U.S. AC05-960R22464. Accordingly, the U.S. Government retains a nonalty-free license to publish or reproduce the published form of this contribution, or allow others to do so, for U.S. Government purposes." 


\section{DISCLAIMER}

This report was prepared as an account of work sponsored by an agency of the United States Government. Neither the United States Government nor any agency thereof, nor any of their employees, make any warranty, express or implied, or assumes any legal liability or responsibility for the accuracy, completeness, or usefulness of any information, apparatus, product, or process disclosed, or represents that its use would not infringe privately owned rights. Reference herein to any specific commercial product, process, or service by trade name, trademark, manufacturer, or otherwise does not necessarily constitute or imply its endorsement, recommendation, or favoring by the United States Government or any agency thereof. The views and opinions of authors expressed herein do not necessarily state or reflect those of the United States Government or any agency thereof. 


\section{DISCLAIMER}

Portions of this document may be illegible in electronic image products. Images are produced from the best available original document. 


$$
\mathrm{S}_{\mathrm{p}}^{\exp }=\frac{\mathrm{T}_{1 / 2}^{\text {calc }}}{\mathrm{T}_{1 / 2}^{\exp }}
$$

The theoretical spectroscopic factor $\left(\mathrm{S}_{\mathrm{p}}^{\text {th }}\right.$ ) can be calculated via an independent quasi-particle BCS approximation [2]. The spectroscopic factor is given by:

$$
\mathrm{S}_{\mathrm{p}}^{\text {th }}=\mathrm{u}_{\mathrm{j}}^{2}
$$

where $u_{\mathrm{j}}^{2}$ is the probability that the proton orbital $(\mathrm{n} \ell \mathrm{j})$ is empty in the daughter nucleus.

The calculations [3] predicting the ground-state deformation of the isotopes reveal that there is a region of high prolate deformation between $Z=50$ and 67 , and then a region of relatively low deformation between $Z=69$ to 82. The effect of deformation on the rate of quantum tunneling is a subject of great interest. To gain insight, however, one needs to understand both spherical and deformed proton emission rates. We can therefore divide proton emission from nuclei with $Z>50$ into two categories: deformed and spherical proton emission.

While calculated values of $S_{p}$ agree quite well for $75 \leq Z \leq 81$, the comparison is rather inconclusive for nuclei with $69 \leq Z \leq 71$, because the known proton emitters in this region have either large uncertainties in their measured branching ratios (as is the case for ${ }^{147} \mathrm{Tm}: 15(5) \%$ [4]), or the $\beta$-decay branch is unknown $\left({ }^{146} \mathrm{Tm}[5]\right.$ and ${ }^{150,151} \mathrm{Lu} \mathrm{[6])} \mathrm{and} \mathrm{is} \mathrm{estimated} \mathrm{from} \mathrm{the} \mathrm{gross} \beta$-decay theory of Takahashi et al [7]. If the estimate is in error by a factor of 2 or more, its use would result in a corresponding uncertainty in the spectroscopic factor. These nuclei lie to the neutron-deficient side of the $\mathrm{N}=\mathbf{8 2}$ closed shell, so that the major competition with proton emission is $\beta^{+}$ rather than $\alpha$ decay. Because $\beta$-decay branching ratios are difficult to measure accurately, large uncertainties exist in the corresponding partial proton half-lives. With this in mind we have concentrated on those proton emitters in this region that were predicted to have half-lives far too short for beta decay to compete with the expected proton emission.

Until recently, the only known ground state proton emitters considered to be deformed were ${ }^{109} \mathrm{I}[8]$ and $112,113 \mathrm{Cs}[8,9]$. These isotopes exhibited longer half-lives than what would have been expected from a simple spherical model. Calculations by Bugrov and Kadmenskii [10] were able to reproduce the experimental data by using a deformation of $\beta_{2} \sim 0.15$ in the parent nucleus ${ }^{109} \mathrm{I}$ and $\beta_{2} \sim 0.21$ for ${ }^{113} \mathrm{Cs}$. More recently, Davids et al. [11] have observed proton emission from the ground state of ${ }^{141} \mathrm{Ho}$, with a proton energy of $1169(8) \mathrm{keV}$ and halflife of 4.2(4) ms. They showed that the data do not agree with spherical WKB predictions but rather with calculations that take deformation into account. In this work, we present new data on ${ }^{141}$ Ho confirming the earlier measurement, and report for the first time proton emission from an isomeric state in ${ }^{141} \mathrm{Ho}$, along with proton emission from ${ }^{140} \mathrm{Ho}$.

\section{Experimental Setup}

The experiments presented here were performed at The Oak Ridge Holifield Radioactive Ion Beam Facility (HRIBF), using the $25 \mathrm{MV}$ Tandem Accelerator. After bombardment on the appropriate target, recoil nuclei of interest were separated spatially according to their mass/charge (A/Q) values through the HRIBF Recoil Mass Spectrometer (RMS) $[12,13,14]$. A gas-filled position sensitive avalanche counter (PSAC) at the focal plane was used to identify the A/Q of the recoils. In reactions similar to those presented herein, the RMS transmission efficiency for the central ion has been determined to be $3-4 \%$ for a typical production target of $0.5-1.0 \mathrm{mg} / \mathrm{cm}^{2}[15]$. Following the PSAC, the central ions were implanted into a $\sim 60-\mu \mathrm{m}$ thick double-sided silicon strip detector (DSSD) [16] with 40 horizontal and 40 vertical strips. This strip arrangement results in a total of 1600 pixels, each acting as an individual detector. Depending on the settings of the RMS, either one or two masses were directed onto the DSSD. When two masses are put on the detector, mass identification is made by the relative $x$-position of the recoils on the DSSD. 
For each event in the DSSD, the time (from a continuously running clock), energy, and event type (recoil or decay, depending on whether it is in coincidence with the PSAC or not) were recorded. By using this time information, the half-life of the decaying nuclide could be determined. The shortest decay observable with our setup is determined by the flight time through the RMS (typically $\sim 2 \mu \mathrm{s}$ ) and the recovery time of the amplifiers after overload due to the implantation of the high-energy recoil. The overload effect causes decay events which occurs shortly $(<\sim 30 \mu \mathrm{sec})$ after the implantation to have a slightly worse energy resolution because each amplifier's response is somewhat different. Individual strips were gain-matched in software through the use of external alpha sources, and internally from ${ }^{147} \mathrm{Tm}[17],{ }^{113} \mathrm{Cs}[8]$ and ${ }^{109} \mathrm{I}[8]$ protons produced in reactions of ${ }^{54} \mathrm{Fe}$ and ${ }^{58} \mathrm{Ni}$ beams on ${ }^{58} \mathrm{Ni}$ and ${ }^{92} \mathrm{Mo}$ targets.

$$
\text { New Spherical Proton Emitters: }{ }^{145} \mathrm{Tm},{ }^{151 \mathrm{~m}_{\mathrm{Lu}} \text { and }}{ }^{150 \mathrm{~m}_{\mathrm{Lu}}}
$$

In the region of $64<\mathrm{Z}<82$, the orbitals $0 \mathrm{~h}_{11 / 2}, 1 \mathrm{~d}_{3} / 2$, and $2 \mathrm{~s} 1 / 2$ are expected to lie close together near the Fermi surface. Of these orbitals, the $d_{3 / 2}$ state can mix with the $s_{1 / 2}$ state coupled to the $2^{+}$phonon of the even-even core excitation. The $h_{11 / 2}$ state is expected, however, to have rather small admixtures. Therefore to rigorously test the predictive power of the BCS-based description of spectroscopic factors, precise $S_{p}^{\exp }$ values for $\pi \mathrm{h}_{11 / 2}$ emitters are essential. With this in mind, we investigated the proton decay of ${ }^{145} \mathrm{Tm}$.

Thulium-145 [18] was produced via the ${ }^{92} \mathrm{Mo}\left({ }^{58} \mathrm{Ni}, \mathrm{p} 4 \mathrm{n}\right)$ reaction. A $0.91-\mathrm{mg} / \mathrm{cm}^{2}$ thick target of ${ }^{92} \mathrm{Mo}$ ( $97 \%$ enrichment) was bombarded with $315-\mathrm{MeV}{ }^{58} \mathrm{Ni}$ ions ( $307 \mathrm{MeV}$ at the target mid-point) extracted from the HRIBF Tandem Accelerator, with an average beam current on target of $\sim 15$ pnA over a period of 50 hours. Figure 1(a) shows the total decay spectrum accumulated in the ${ }^{58} \mathrm{Ni}+{ }^{92} \mathrm{Mo}$ irradiation. One observes $\alpha$-decay peaks of nuclei produced in reactions on target isotopic impurities whose $A / Q$ value is similar to that of the central ion. The low energy part of the spectrum is dominated by a broad distribution of "escape" events resulting from $\alpha$ decays in the backward direction, i.e., only part of the energy is deposited in the DSSD. Also visible is a peak at $1.12 \mathrm{MeV}$ which we attribute to protons from ${ }^{146} \mathrm{Tm}[5,19]$, and which provided an internal energy calibration. Figure 1 (b) shows only those events that had a time between implantation and decay of $\leq 50 \mu \mathrm{s}$. In this spectrum, a peak with an energy of $1.728(10) \mathrm{MeV}$ is clearly seen. We assign it to the proton decay of ${ }^{145} \mathrm{Tm}$ as no other nucleus with $\mathrm{A}$ $=145$ and $Z<69$ is proton unbound [20]. Since all the events in this peak came at very short times after recoil implantation, the energy resolution is poorer than what is normally observed for decays with longer half-lives. By correcting for the overload effect of the amplifiers, (based on the measured proton spectra of ${ }^{113} \mathrm{Cs} ; \mathrm{T}_{1 / 2}=18 \mu \mathrm{s}$ ) the resolution of this peak was improved as shown in Fig. 1(c). If the overall detection efficiency of the RMS is taken to be $3 \%$ (for this reaction), the production cross section for ${ }^{145} \mathrm{Tm}$ is estimated to be $500 \mathrm{nb}$.

As mentioned above, half-life information can be obtained by correlating the times of a recoil and the next decay event in a given pixel. The resulting half-life for the ${ }^{145} \mathrm{Tm}$ proton peak is $3.5(10) \mu \mathrm{s}$. A WKB approximation calculation for protons emitted from the $0 \mathrm{~h}_{11 / 2}(\Delta \ell=5), 1 \mathrm{~d}_{3 / 2}(\Delta \ell=2)$, and $2 \mathrm{~s}_{1 / 2}(\Delta \ell=0)$ orbitals of ${ }^{145} \mathrm{Tm}$ was performed with the experimental $\mathrm{E}_{\mathrm{p}}$ as input. The optical potential was taken from the real part of the optical potential of Becchetti and Greenlees [21]. The calculated half-lives are $1.8_{-0.2}^{+0.3} \mu \mathrm{s}, 0.7(1) \mathrm{ns}$ and $80(12) \mathrm{ps}$, respectively for $\Delta \ell=5,2$, and 0 . Table 1 compares these values to the experimental value of $3.5(10) \mu \mathrm{s}$ and clearly indicates a $\Delta \ell=5$ transfer and thus an $0 \mathrm{~h}_{11 / 2}$ assignment for the parent proton state which, based on level systematics in this mass region, is probably the ground state in ${ }^{145} \mathrm{Tm}$. Taking the WKB value of $1.8 \mu$ s for the theoretical half-life results in a spectroscopic factor of $0.51(16)$ for this $0 h_{11 / 2}$ emitter. The error should be considered as a lower limit since uncertainties due to the optical potential are not included. This value is consistent with an overall spherical description for this nucleus, and agrees well with the value of 0.64 for the $S_{p}^{\text {th }}$ obtained by Åberg et al [2] using the BCS approximation. This work represents the first $\mathrm{S}_{\mathrm{p}}^{\exp }$ value with error bars small enough to be meaningful for the $0 \mathrm{~h}_{11 / 2}$ proton emission from odd-mass Tm isotopes.

The lutetium isotopes were among the first nuclei where proton emission was observed. In fact ${ }^{151} \mathrm{Lu}$ was the first ground state proton emitter discovered [22]. However, for both the cases of ${ }^{151} \mathrm{Lu}$ and ${ }^{150} \mathrm{Lu}$, only one high-spin proton-emitting isomer has been observed. Neighboring nuclei however, have been observed to emit a 
TABLE 1. Comparison of the observed proton half-lives with values calculated using the spherical WKB approximation.

\begin{tabular}{|c|c|c|c|c|c|}
\hline \multirow[t]{2}{*}{ species } & \multirow[t]{2}{*}{$\mathrm{E}_{\mathrm{p}}(\mathrm{MeV})$} & \multirow[t]{2}{*}{$\operatorname{Exp} T_{1 / 2}$} & \multicolumn{3}{|c|}{ calculated $\mathrm{T}_{1 / 2}(\mathrm{WKB})$} \\
\hline & & & $\mathrm{Oh}_{11 / 2}(\Delta \ell=5)$ & $1 \mathrm{~d}_{3 / 2}(\Delta \ell=2)$ & $2 \mathrm{~s}_{1 / 2}(\Delta \ell=0)$ \\
\hline${ }^{145} \mathrm{Tm}$ & $1.728(10)$ & $\mathbf{3 . 5 ( 1 0 ) \mu s}$ & $1.8_{-0.2}^{+0.3} \mu s$ & $0.7(1) \mathrm{ns}$ & $80(12) \mathrm{ps}$ \\
\hline $151^{\mathrm{m}} \mathrm{Lu}$ & $1.310(15)$ & $16(1) \mu s$ & $13_{-4}^{+6} \mathrm{~ms}$ & $4.9_{-1.4}^{+2.0} \mu s$ & $0.55_{-0.14}^{+0.28} \mu \mathrm{s}$ \\
\hline${ }^{150} \mathrm{Lu}$ & $1.263(4)^{*}$ & $49(5) \mathrm{ms}$ & $36(3) \mathrm{ms}$ & $13(1) \mu \mathrm{s}$ & $1.5(1) \mu \mathrm{s}$ \\
\hline $150 \mathrm{~m}_{\mathrm{Lu}}$ & $1.290_{-30}^{+20}$ & $30_{-10}^{+40} \mu s$ & $19_{-7}^{+20} \mathrm{~ms}$ & ${ }_{-3}^{+7} \mu s$ & $0.7_{-0.2}^{+1.5} \mu \mathrm{s}$ \\
\hline $141^{\mathrm{m}} \mathrm{Ho}$ & $1.230(20)$ & $8.4_{-1.8}^{+3.1} \mu s$ & $7_{-3}^{+4} \mathrm{~ms}$ & $2(1) \mu s$ & $0.2(1) \mu \mathrm{s}$ \\
\hline${ }^{141} \mathrm{Ho}$ & $1.169(8)^{\dagger}$ & $3.9(5) \mathrm{ms}$ & $32(7) \mathrm{ms}$ & $8.6_{-1.6}^{+1.9} \mu \mathrm{s}$ & $0.89 \begin{array}{l}+0.20 \\
-0.16\end{array} \mu \mathrm{s}$ \\
\hline${ }^{140} \mathrm{Ho}$ & $1.086(10)$ & $6.1 \stackrel{+3.1}{-1.5} \mathrm{~ms}$ & $320_{-80}^{+100} \mathrm{~ms}$ & $81 \stackrel{+26}{-20} \mu s$ & ${ }_{-2}^{+3} \mu s$ \\
\hline $\begin{array}{ll}* & \text { Energy } \\
\dagger & \text { Energy }\end{array}$ & $\begin{array}{l}\text { m Ref } 6 . \\
\text { m Ref. } 11\end{array}$ & & & & \\
\hline
\end{tabular}

proton from both a high- and low-spin isomer. As with the Tm isotopes, it is expected that the $0 \mathrm{~h}_{11 / 2}, 1 \mathrm{~d}_{3 / 2}$, and $2 s_{1 / 2}$ orbitals lie close to the ground state. With this in mind, we reinvestigated the proton decays of ${ }^{151} \mathrm{Lu}$ and ${ }^{150} \mathrm{Lu}$.

Lutetium-151 was produced via the ${ }^{96} \mathrm{Ru}\left({ }^{58} \mathrm{Ni}, \mathrm{p} 2 \mathrm{n}\right)$ reaction with a beam energy of $266 \mathrm{MeV}$. Figure 2 (a) shows the spectrum obtained from the above reaction with a constraint on the time between recoil and decay of $\leq$ $500 \mathrm{~ms}$. A further constraint on the time of $\leq 50 \mu \mathrm{s}$ is shown in Fig. 2(b). It clearly shows a peak at 1310(15) keV which we attribute to the proton decay of an isomer located at an energy of $80(15) \mathrm{keV}$ [23] above the previously known $h_{11 / 2}$ ground state. The half-life associated with this new radioactivity is $16(1) \mu \mathrm{s}$. A WKB approximation calculation for protons emitted from the $0 \mathrm{~h}_{11 / 2}(\Delta \ell=5), 1 \mathrm{~d}_{3 / 2}(\Delta \ell=2)$, and $2 \mathrm{~s}_{1 / 2}(\Delta \ell=0)$ orbitals of ${ }^{151} \mathrm{Lu}$ was performed with the experimental $\mathrm{E}_{\mathrm{p}}$ as input (see table 1). As can be seen from the table, the calculated value that matches most closely is the value of $4.9_{-1.4}^{+2.0} \mu \mathrm{s} \mu \mathrm{s}$ for emission from the $\mathrm{d}_{3 / 2}$ state, with resulting spectroscopic factor of $0.31_{-0.09}^{+0.12}$. This value is low when compared to the calculated $u_{j}^{2}$ of 0.74 [24], which is typical of the $d_{3 / 2}$ proton emitters (see Fig 4 ).

For the case of the odd-odd nucleus ${ }^{150} \mathrm{Lu}$, one might expect that it also exhibits two proton emitting isomers, similar to the situation for ${ }^{146} \mathrm{Tm}$. If one considers coupling the neutron quasi-particles to the proton orbitals, the resulting lowest proton unbound states are $\pi \mathrm{h}_{11 / 2} \otimes v \mathrm{~h}_{11 / 2}, \pi \mathrm{h}_{11 / 2} \otimes v \mathrm{~d}_{3 / 2}, \pi \mathrm{d}_{3 / 2} \otimes v \mathrm{~d}_{3 / 2}$ and $\pi \mathrm{d}_{3 / 2} \otimes \mathrm{vh}_{11 / 2}$. The known [6] isomer is attributed to arise from decay out of the $5^{-}$or $6^{-}$state to the $1 / 2^{+}$or $3 / 2^{-}$ proton decaying state in the ${ }^{149} \mathrm{Yb}$ daughter. During our reinvestigation of this nucleus, we discovered a second short-lived proton emitting state in ${ }^{150} \mathrm{Lu}$. We produced ${ }^{150} \mathrm{Lu}$ via the ${ }^{96} \mathrm{Ru}\left({ }^{58} \mathrm{Ni}, \mathrm{p} 3 \mathrm{n}\right)$ reaction at a beam energy of $292 \mathrm{MeV}$ (at the target midpoint). Figure 2(c) shows the total decay spectrum with a time constraint between decay and recoil of $\leq 100 \mathrm{~ms}$. The known ${ }^{150} \mathrm{Lu}$ proton peak is clearly shown at $1261 \mathrm{keV}$. Figure $2(\mathrm{~d})$ shows the same spectrum with a further constraint on the time of $\leq 100 \mu \mathrm{s}$. In this figure a higher energy peak of 1290(30) 
$\mathrm{keV}$ is observed, which we attribute to the decay of a short-lived isomer of ${ }^{150} \mathrm{Lu}$. The half-lives of these two transitions resulting from this measurement are $49(5) \mathrm{ms}$ (compared to $35(10) \mathrm{ms}$ from Ref. [6]), and $30_{-10}^{+40} \mu \mathrm{s}$ for the new isomer. Ytterbium-149 is expected to have a ground-state spin of $1 / 2^{+}$or $3 / 2^{+}$and an isomer of $11 / 2^{-}$ similar to the structure observed for ${ }^{151} \mathrm{Yb}$ [25]. We therefore propose that the new proton transition from ${ }^{150} \mathrm{Lu}$ is the decay of the isomeric $\pi d_{3 / 2} \otimes v d_{3 / 2}$ state to a $v d_{3 / 2}$ state (probably the ground state) in ${ }^{149} \mathrm{Yb}$.

The known experimental (including this work) values for the spherical $\mathrm{Sp}$ are compared with the theoretical ones in Fig. 4 (a) for $h_{11 / 2}$ and 4 (b) for $d_{3 / 2}$. Comparing the two figures, one can see that for $h_{11 / 2}$ values, the agreement between experimental and theoretical $S_{p}$ values is fairly good (except for the large error bars), while the experimental $\mathrm{d}_{3 / 2}$ values are consistently too low compared to the theoretical values. This probably indicates that the $d_{3 / 2}$ proton emitting states have a significant amount of mixing with other configurations such as $2^{+} \otimes \pi s_{1 / 2}$.

\section{New Deformed Proton Emitters: ${ }^{140} \mathrm{Ho}$ and ${ }^{141 \mathrm{~m}} \mathrm{Ho}$}

Deformation has been predicted [26] to increase substantially along the proton drip-line between the $\operatorname{Tm}\left(\beta_{2}\right.$ $\leq 0.15)$ and the Ho isotopes $\left(\beta_{2} \geq 0.25\right)$. Therefore, the study of the proton decay of the Ho isotopes will give us some insight into the effects of large deformation on the tunneling process. Previous to this study, the only highly deformed proton emitters known were ${ }^{141} \mathrm{Ho}$ and ${ }^{131} \mathrm{Eu}$ [11]. Our investigations have led to the discovery of proton decay from a short-lived isomer of ${ }^{141} \mathrm{Ho}$ and from a new isotope ${ }^{140} \mathrm{Ho}$ [27]. Holmium-140 and ${ }^{141} \mathrm{Ho}$ were produced via the ${ }^{92} \mathrm{Mo}\left({ }^{54} \mathrm{Fe}, \mathrm{p} 5 \mathrm{n}\right)$ and ${ }^{92} \mathrm{Mo}\left({ }^{54} \mathrm{Fe}, \mathrm{p} 4 \mathrm{n}\right)$ reactions, respectively, using $315-\mathrm{MeV}{ }^{54} \mathrm{Fe}$ ions extracted from the HRIBF Tandem Accelerator. In this experiment, ions of mass 140 were deposited on one side (strips \# 2040) of the DSSD, and mass 141 were deposited on the other (\# 1-19).

Figure 3(a) shows the decay spectrum obtained from the above reaction with $A=141$, and a time constraint between decay and recoil of $T \leq 16 \mathrm{~ms}$. In this spectrum a peak is observed at $\sim 1170 \mathrm{keV}$ with a rather broad shoulder on the high energy side. Further constraints on the time reveals that it consists of two peaks: the known proton peak from ${ }^{141} \mathrm{Ho}$, and a new proton transition from a short-lived isomer of ${ }^{141} \mathrm{Ho}$. In figure $3(\mathrm{~b})$ we

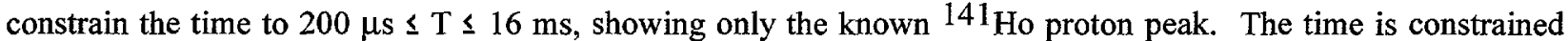
in Fig. 3(c) to those events of $T \leq 50 \mu$ s. In this spectrum, a higher energy peak is observed. The energies and half-lives of these two peaks are $\mathrm{E}_{\mathrm{p}}=1230(20) \mathrm{keV}, \mathrm{T}_{1 / 2}=8.4_{-1.8}^{+3.1} \mu$ s for the new isomer of ${ }^{141} \mathrm{Ho}$ and $\mathrm{E}_{\mathrm{p}}=$ $1169(8) \mathrm{keV}$ (this peak was used as a calibration point), $\mathrm{T}_{1 / 2}=3.9(5) \mathrm{ms}$ for the previously known proton arising from the ground state of ${ }^{141} \mathrm{Ho}$, (which compares well with the literature value 4.2(4) ms [11]). Figure 3(d) shows the decay spectrum from mass 140 with the time constraint of $200 \mu \mathrm{s} \leq \mathrm{T} \leq 16 \mathrm{~ms}$. Here, two lines are observed; one from the ${ }^{141} \mathrm{Ho}$ ground state (due to overlap of the tail of mass 141 ), and one from the new isotope ${ }^{140} \mathrm{Ho}$, with values of $\mathrm{E}_{\mathrm{p}}=1086(10) \mathrm{keV}$, and $\mathrm{T}_{1 / 2}=6.1_{-1.5}^{+3.1} \mathrm{~ms}$.

As can be seen from Table 1, the spherical WKB approach gives values which can not easily explain the experimental half-lives of the Ho isotopes. In Ref. 11 Davids et al., demonstrate that the ${ }^{141}$ Ho ground-state decay can be explained using the formalism developed by Bugrov and Kadmenskii [28], resulting in a good fit for $\beta_{2} \sim 0.3$ for proton emission from the $\pi 7 / 2^{-}$[523] state originating from the $h_{11 / 2}$ orbital. Our calculations [29], which minimize the energies of the ${ }^{141}$ Ho states in deformation space, indicate that the $\pi 1 / 2^{+}[411]$ and the $\pi 7 / 2^{-}$[523] have nearly the same energy and deformation $\left(\beta_{2} \sim 0.27\right.$ and $\left.\beta_{4} \sim-0.07, \beta_{6} \sim 0.01\right)$. The next excited state, $\pi 5 / 2^{-}$ [532], is predicted to be $250 \mathrm{keV}$ higher. Proton emission from the $\pi 1 / 2^{+}[411]$ and $\pi 7 / 2^{-}$[523] states to the ground state of ${ }^{140} \mathrm{Dy}$ would have $\Delta \ell$ equal to 0 and 3 respectively. The spherical WKB approach predicts the proton $T_{1 / 2}$ values to be $0.2 \mu \mathrm{s}$ and $1.7 \mu \mathrm{s}$, respectively, overestimating the proton emission probability. The proton decay rate from the deformed states is clearly reduced due to the complex structure of deformed wave function and tunneling through the deformed Coulomb barrier $[11,28,29]$. Based on the measured half-lives, we assign $\pi 7 / 2^{-}$ [523] to the ground state, and $\pi 1 / 2^{+}[411]$ of the $60(20) \mathrm{keV}$ isomer of ${ }^{141} \mathrm{Ho}$.

Proton emission from the odd-odd nucleus ${ }^{140} \mathrm{Ho}$ is more complicated as one has to consider both the proton and neutron quasi-particles. The aforementioned calculations predict that the $\pi 7 / 2^{-}[523] \otimes v 9 / 2^{-}[514]$, $\pi 7 / 2[523] \otimes v 5 / 2^{+}[402], \pi 1 / 2^{+}[411] \otimes v 9 / 2^{-}[514]$ and $\pi 1 / 2^{+}[411] \otimes v 5 / 2^{+}[402]$ levels all lie within $20 \mathrm{keV}$ of 
the ground state. Based on information from the ${ }^{141}$ Ho proton decay, the $\pi 7 / 2^{-}[523]$ orbital coupled with either the $v 9 / 2^{-}[514]$ or $v 5 / 2^{+}[402]$ orbital can be assigned to the observed proton-emitting state in ${ }^{140} \mathrm{Ho}$.

\section{ACKNOWLEDGEMENTS}

* UNIRIB is a consortium of universities, Oak Ridge Associated Universities, and is supported by them and the U.S. Department of Energy under contract No. DE-AC05-76OR00033 with the Oak Ridge Associated Universities. ORNL is managed by Lockheed Martin Energy Research under contract number DE-AC05-96OR22464 with the U.S. Department of Energy. ZJ and MK are partially supported by KBN, Poland, contract KBN 2 P03B 03913.

\section{REFERENCES}

1. M. H. McFarlane, and J. B. French, Rev. Mod. Phys. 32, 567 (1960).

2. S. Åberg, P. B. Semmes, and W. Nazarewicz, Phys. Rev. C 56, 1762, (1997).

3. P. Möller, et al., At. Data Nucl. Data Tables 59, 185 (1995).

4. K. S. Toth, D. C. Sousa, P. A. Wilmarth, J. M. Nitschke, and K. S. Vierinen, Phys. Rev. C. 471804 (1993).

5. K. Livingston, P. J. Woods, T. Davinson, N. J. Davis, S. Hoffman, A. N. James, R. D. Page, P. J. Sellin, and A. C. Shotter, Phys. Lett. 312B 46, (1993).

6. P. J. Sellin, P. J. Woods, T. Davinson, N. J. Davis, K. Livingston, R. D. Page, A. C. Shotter, S. Hoffman, and A. N. James, Phys Rev C 47, 193 (1993).

7. K. Takahashi, M. Yamada and T. Kondoh, At. Data Nucl. Data Tables 12, 101 (1973).

8. A. Gillitzer, T. Faestermann, K. Hartel, P. Kienle, and E. Nolte, Z. Phys. A326, 107 (1987).

9. R. D. Page, P. J. Woods, R. A. Cunningham, T. Davinson, N. J. Davis, S. Hoffman, A. N. James, K. Livingston, P. J. Sellin, and A. C. Shotter, Phys. Rev. Lett. 68, 1287 (1992).

10. V. P. Bugrov and S. G. Kadmenskii, Sov. J. Nucl. Phys. 49, 967 (1989)..

11. C. N. Davids, P.J. Woods, D. Seweryniak, A. A. Sonzogni, J. C. Batchelder, C. R. Bingham, T. Davinson, D.J. Henderson, R. J. Irvine, G. L. Poli, J. Uusitalo, and W. B. Walters, Phys. Rev. Lett. 80, 1849 (1998)

12. T. M. Comier, J. D. Cole, J. H. Hamilton and A. V. Ramayya, Nucl. Instrum. Methods Phys. Res. A297, 199, (1990).

13. J. D. Cole, T. M. Cormier, J. H. Hamilton and A. V. Ramayya, Nucl. Instrum. Methods Phys. Res. B70, 343 (1992).

14. C. J. Gross, Y. A. Akovali, M. J. Brinkman, J. W. Johnson, J. Mas, J. W. McConnell, W. T. Milner, D. Shapira, and A. N. James, Application of Accelerators in Research and Industry, AIP conference proceedings 392, Woodbury, NY, Vol 1, (1997) p 401.

15. C. J. Gross, private communication.

16. P. J. Sellin, P. J. Woods, D. Branford, T. Davinson, N. J. Davis, D. G. Ireland, K. Livingston, R. D. Page, A. C. Shotter, S. Hofmann, R. A. Hunt, A. N. James, M. A. C. Hotchkis, M. A. Freer, S. L. Thomas, Nucl. Instrum. Methods Phys. Res. A311, 217 (1992).

17. P. O. Larsson, T. Batsch, R. Kirchner, O. Klepper, W. Kurcewicz, E. Roeckl, D. Schart, W. F. Feix, G. Nyman, P. Tidemand-Petersson, Z. Phys. A314, 9 (1983).

18. J. C. Batchelder, C. R. Bingham, K. Rykaczewski, K. S. Toth, T. Davinson, J. A. McKenzie, P. J. Woods, J. H. Hamilton, W. B. Walters, E. F. Zganjar, C. Baktash, T. N. Ginter, J. Greene, C. J. Gross, J. Mas, J. McConnell, S. D. Paul, D. Shapira, X. Xu, and C. -H. Yu, Phys Rev C 57, R1042 (1998).

19. P. J. Woods, T. Davinson, N. J. Davis, S. Hoffman, A. N. James, K. Livingston, R. D. Page, P. J. Sellin, and A. C. Shotter, Nucl. Phys. A553, (1993).

20. G. Audi and H. Wapstra, Nucl. Phys. A565, 66 (1993).

21. F. D. Becchetti, Jr. and G. W. Greenlees, Phys. Rev. 182, 1190 (1969).

22. S. Hoffman, W. Reisdorf, G. Münzenberg, F. P. Hessberger, J. R. H. Schneider, and P. Armbruster, Z. Phys. A305, 111 (1982).

23. C. R. Bingham, J. C. Batchelder, K. Rykaczewski, K. S. Toth, C. -H. Yu, T. N. Ginter, C. J. Gross, R. Grzywacz, M. Karney, S. H. Kim, B. D. MacDonald, J. Mas, J. W. McConnell, J. Szerypo, and W. Weintraub, to be published.

24. W. Nazarewicz, private communication.

25. E. Nolte, G.Korschinek, Ch. Setzensack Z. Phys. A309, 33 (1982)

26. P. Möller, J. R. Nix, W. D. Myers and j. Swiatecki, At. Data Nucl. Data Tables 59, 185 (1995).

27. K. Rykaczewski, J. C. Batchelder, C. R. Bingham, K. S. Toth, T. Davinson, T. N. Ginter, C. J. Gross, R. Grzywacz, M. Karny, B. D. MacDonald, J. F. Mas, J. W. McConnell, A. Piechaczek, R. C. Slinger, P. J. Woods, E. F. Zganjar, to be published.

28. V. P. Bugrov and S. G. Kadmenskii, Sov. J. Nucl. Phys. 49, 967 (1989).

29. W. Nazarewicz, M. A. Riley, and J. D. Garrett, Nucl. Phys. A512, 61 (1990). 


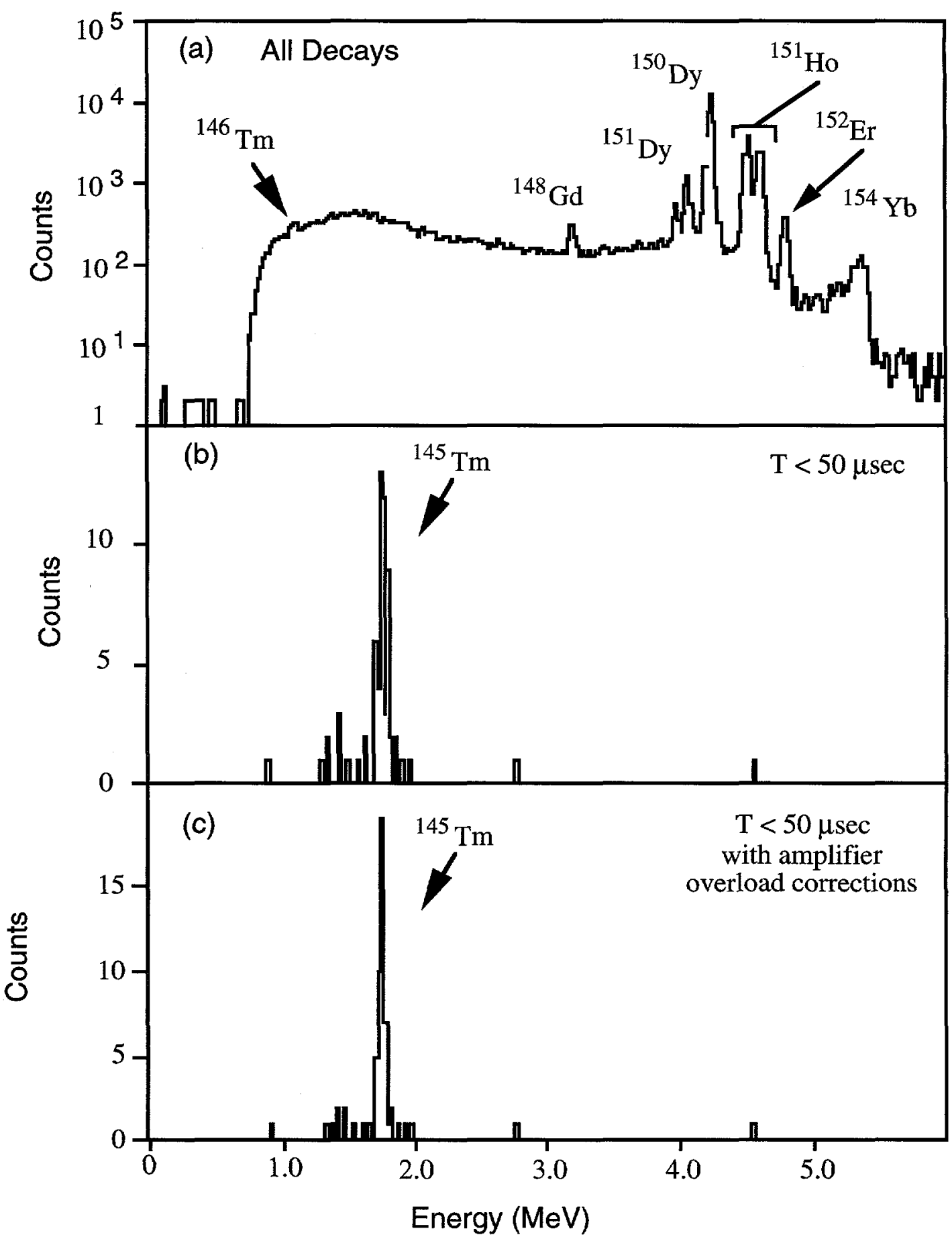

FIGURE 1. (a) Total decay spectrum observed in the DSSD during $307-\mathrm{MeV}{ }^{58} \mathrm{Ni}$ bombardments of ${ }^{92} \mathrm{Mo}$. The $\alpha$ peaks above $3 \mathrm{MeV}$ arise from nuclei formed in reactions on isotopic impurities present in the ${ }^{92} \mathrm{Mo}$ target. (b) The same spectrum gated on $A=145$ recoils, with a time between decay and recoil implantation of $\leq 50 \mu \mathrm{s}$. (c) The spectrum in (b) with corrections to the resolution resulting from the amplifier overload of each strip. 


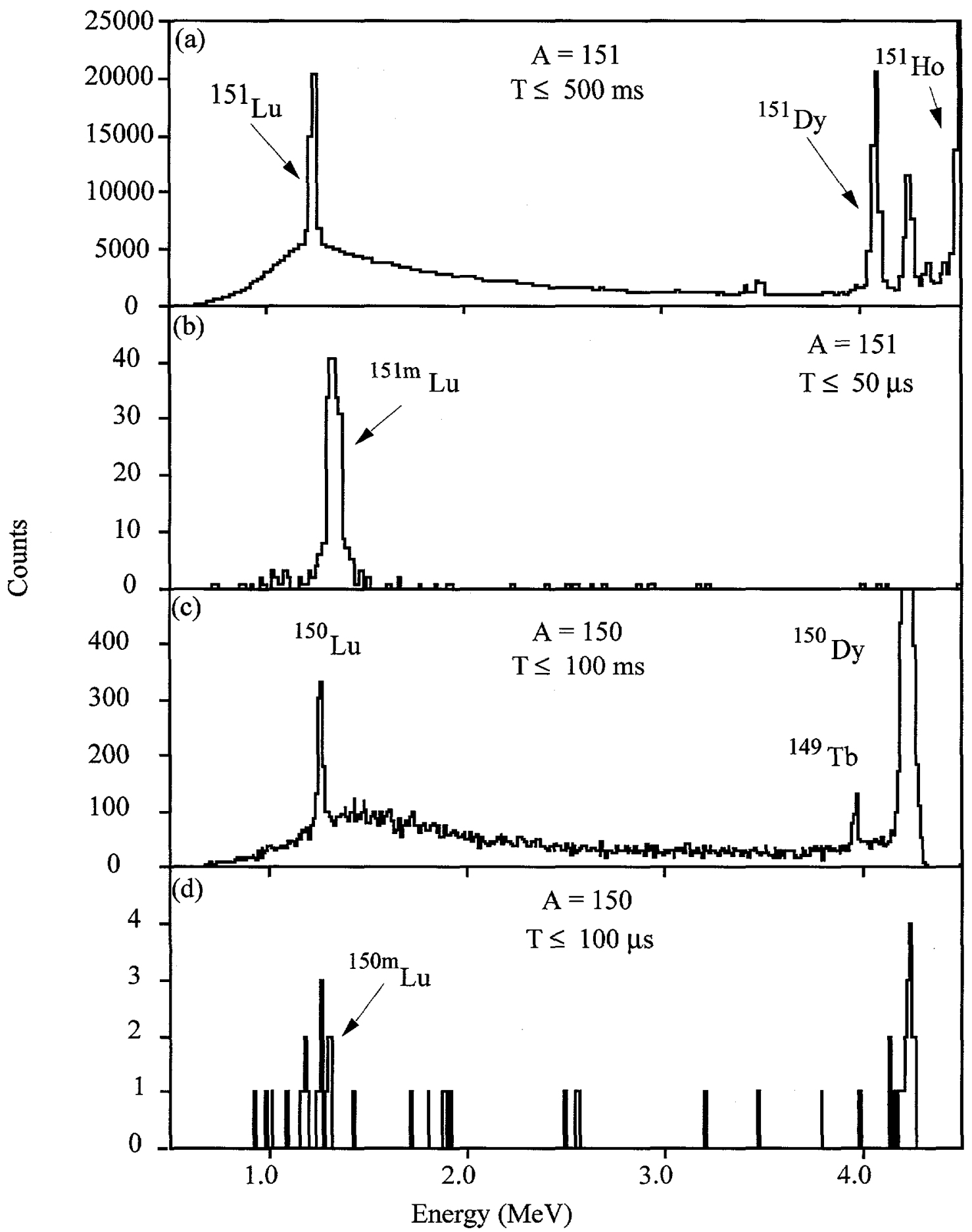

FIGURE 2. (a) Decay spectrum observed during $270-\mathrm{MeV}{ }^{58} \mathrm{Ni}$ bombardments of ${ }^{96} \mathrm{Ru}$, with a time between decay and recoil implantation of $\leq 500 \mathrm{~ms}$. (b) The decay spectrum from (a) with a further time constraint of $\leq 50 \mu \mathrm{s}$.(c) Decay spectrum observed during $292 \mathrm{MeV}{ }^{58} \mathrm{Ni}$ bombardments on ${ }^{96} \mathrm{Ru}$, with a time constraint of $\mathrm{T} \leq 100 \mathrm{~ms}$ between decay and recoil. (d) The same spectrum as (c) with a time constraint of $\mathrm{T} \leq 100 \mu \mathrm{s}$ 


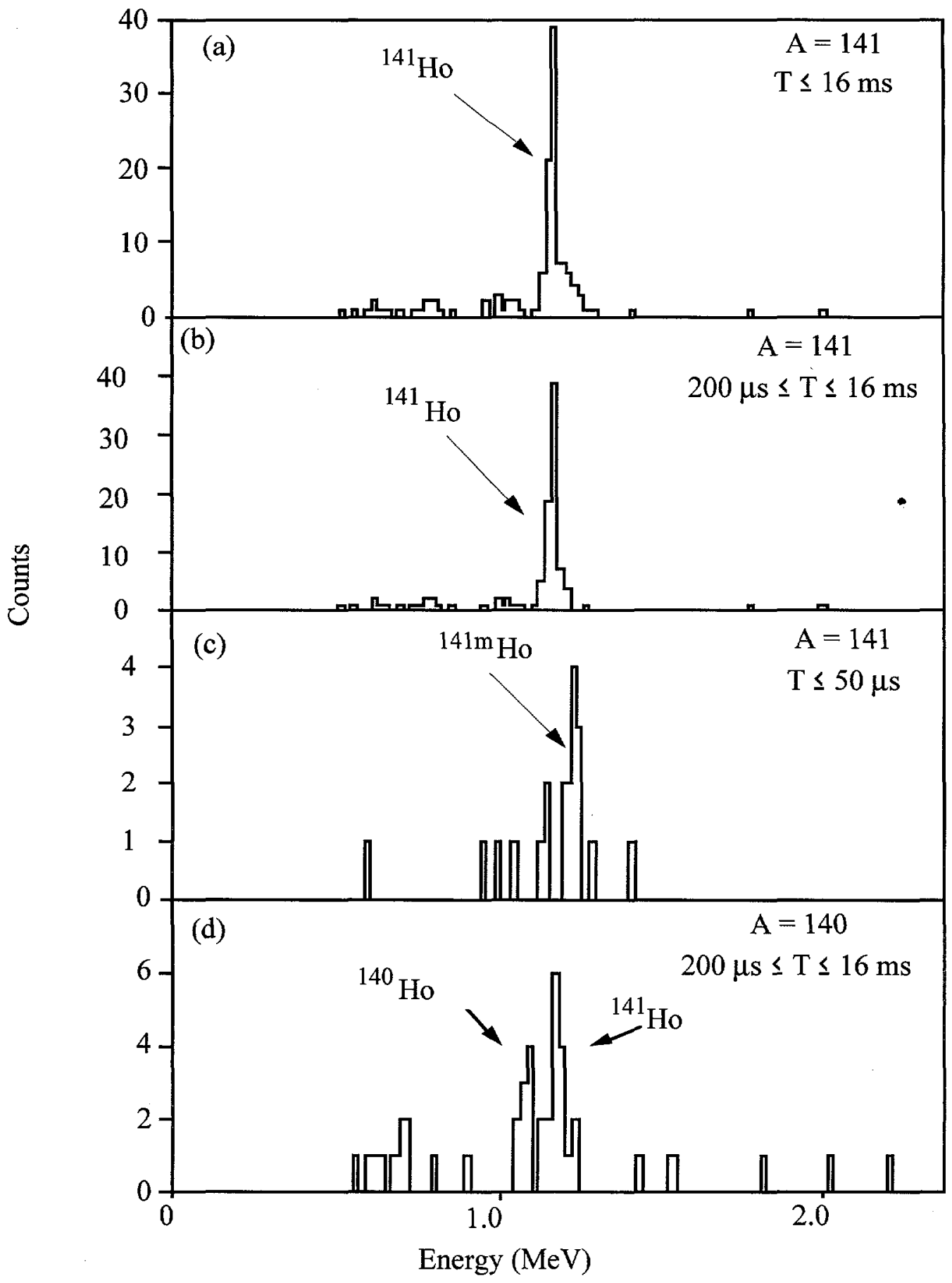

FIGURE 3. (a) Decay spectrum from $315-\mathrm{MeV}{ }^{54} \mathrm{Fe}$ bombardments of ${ }^{92} \mathrm{Mo}$, with $\mathrm{A}=141$, and a time between decay and recoil implantation of $\leq 160 \mathrm{~ms}$. The same spectrum is shown with a time constraint of (b) $200 \mu \mathrm{s} \leq \mathrm{T} \leq 160 \mathrm{~ms}$, and (c) $\mathrm{T} \leq 50 \mu \mathrm{s}$. The decay spectrum with $\mathrm{A}=140$ with a time constraint of $200 \mu \mathrm{s} \leq \mathrm{T} \leq 160 \mathrm{~ms}$ is shown in (d). 


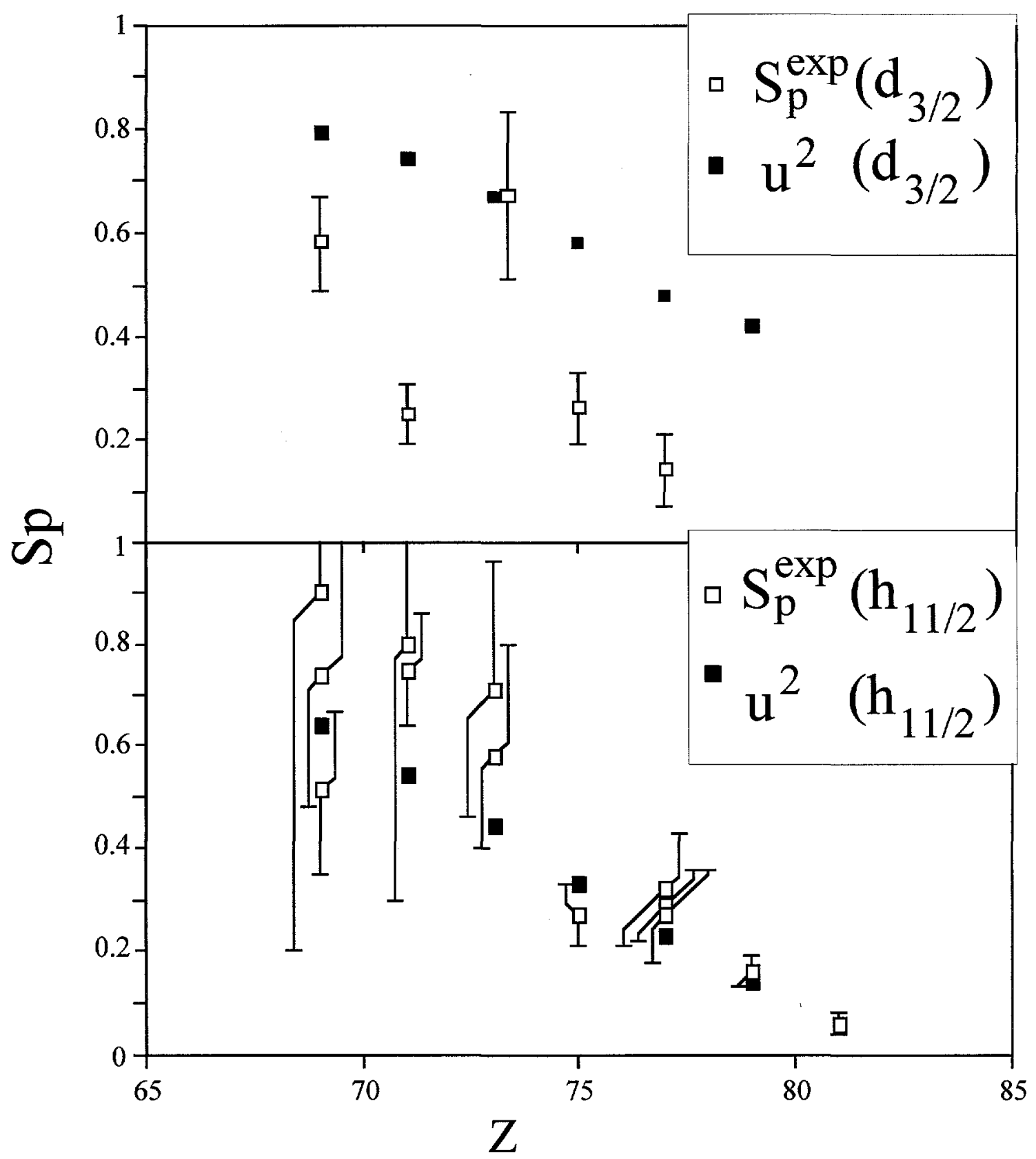

FIGURE 4. Comparison of $S_{p}^{\exp }$ and $u^{2}$ for known proton emitters with $69 \leq Z \leq 81$. Part (a) compares the values for protons emitted form the $\pi \mathrm{d}_{3 / 2}$ orbital, while (b) contains the values for the $\pi \mathrm{h}_{11 / 2}$ orbitals. The values for $\mathrm{u}^{2}$ are taken from reference 2 . 